\title{
Un "país” rico en necesidades: un experimento en el Seminario en Enfermería de Salud Pública (1962)
}

\author{
$A$ “ country" rich in needs: an experiment in Seminario en \\ Enfermeria of Salud Publica (1962)
}

\section{Um "País" rico em necessidades: um experimento no Seminário en Enfermeria de Salud Publica (1962)}

Petite Fleur Custódio da Silva ${ }^{1}$, Aline Dos Santos da Silva², Erika Bicalho de Almeida ${ }^{3}$, Julieta Brites Figueiredo ${ }^{4}$, Vanessa Costa Cavalcanti ${ }^{5}$, Wellington Mendonça Amorin ${ }^{6}$.

${ }^{1}$ Enfermeira, membro do Laboratório de Abordagens Científicas na História da Enfermagem (LACENF), da Universidade Federal do Estado do Rio de Janeiro - UNIRIO. Rua Dr. Xavier Sigaud, 290, sala 308 - URCA, Rio de Janeiro, CEP 222290-180, Brasil. Telefone: (21) 997453155. E-mail: fleurpetite.2@gmail.com.

${ }^{2}$ Mestranda em Enfermagem pelo Programa de Pós-Graduação em Enfermagem (PPGENF) da Escola Alfredo Pinto - Universidade Federal do Estado do Rio de Janeiro (UNIRIO). Membro do Laboratório de Abordagens Científicas na História da Enfermagem (LACENF) e do Laboratório de Pesquisas em História da Enfermagem (LAPHE). Rio de Janeiro - RJ. Brasil. E-mail: line-enf@ hotmail.com.

${ }^{3}$ Doutoranda em Enfermagem pelo Programa de Pós-Graduação em Enfermagem (PPGENF) da Escola Alfredo Pinto - Universidade Federal do Estado do Rio de Janeiro (UNIRIO). Mestre em Educação pela Universidade Católica de Petrópolis (UCP). Membro do Laboratório de Abordagens Científicas na História da Enfermagem (LACENF). Rio de Janeiro - RJ. Brasil. E-mail: ebicalhoenf@hotmail.com.

${ }^{4}$ Doutoranda em Enfermagem pelo Programa de Pós-Graduação em Enfermagem (PPGENF) da Escola Alfredo Pinto - Universidade Federal do Estado do Rio de Janeiro (UNIRIO). Mestre em Educação Profissional em Saúde pela Escola Politécnica de Saúde Joaquim Venâncio (EPSJV) - Fiocruz. Membro do Laboratório de Abordagens Científicas na História da Enfermagem (LACENF).

Rio de Janeiro - RJ. Brasil.E-mail: juliabrites@ig.com.br.

${ }^{5}$ Mestranda em Enfermagem pelo Programa de Pós-Graduação em Enfermagem (PPGENF) da Escola Alfredo Pinto - Universidade Federal do Estado do Rio de Janeiro (UNIRIO). Membro do Laboratório de Abordagens Científicas na História da Enfermagem

(LACENF), da Universidade Federal do Estado do Rio de Janeiro - UNIRIO. E-mail: enfavanessacavalcanti@gmail.com

${ }^{6}$ Doutor em Enfermagem pela UFRJ. Professor Adjunto do Departamento de Enfermagem em Saúde Pública, da Escola de Enfermagem Alfredo Pinto, da Universidade Federal do Estado do Rio de Janeiro (UNIRIO). Líder do Laboratório de Abordagens Científicas na História da Enfermagem (LACENF). Rio de Janeiro - RJ. Brasil. E-mail: amorimw@gmail.com.

Cómo citar este artículo en edición digital: da Silva, P. F. C., da Silva, A. S., de Almeida, É. B., Figueiredo, J. B., Cavalcanti, V. C., \& de Amorim, W. M. (2018). Un "país" rico en necesidades: un experimento en el Seminario en Enfermería de Salud Pública (1962 ). Cultura de los Cuidados (Edición digital), 22(50). Recuperado de http://dx.doi.org/10.14198/cuid.2018.50.05

Correspondencia: Rua Dr. Xavier Sigaud, 290, sala 308 - URCA, Rio de Janeiro, CEP 222290-180, Brasil. Telefone: (21) 997453155. Correo electrónico: fleurpetite.2@gmail.com. Recibido: 20/05/2017; Aceptado: 10/10/2017

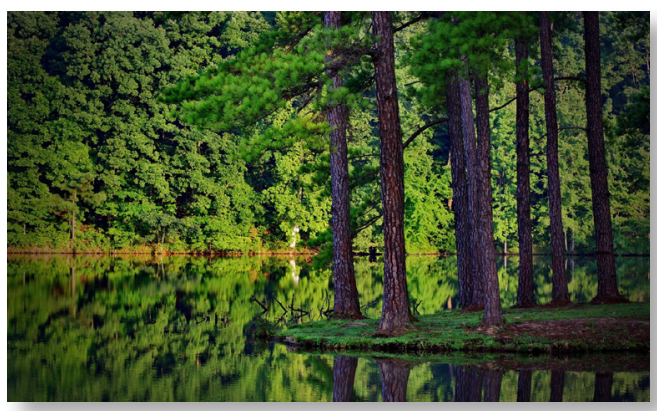

\begin{abstract}
The study aimed to discuss the intended effects on Latin American nurses legitimated by the fictional strategy for the planning of public health nursing. Qualitative research approach based on the historical perspective of the Cultural History of the French historian Roger Chartier, and operationalized in the techni-
\end{abstract}


cal documentation of observation, and as a source publication Tell Del Seminario of Public Health - PAHO categories emerged after analyzing needs, planning and fictional narrative health actions. Result: he met through the fictional narrative power relations, social configurations of class and character and context of nurses in Latin America. It is concluded that the strategy of the seminar enabled the construction of collective and joint proposals from the exchange of experiences between the participating nurses.

Keywords: nursing, history of nursing, public health, health services needs and demand, health policy.

\section{RESUMEN}

El objetivo del estudio fue analizar los efectos previstos en el personal de enfermería de América Latina legitimados por la estrategia de ficción para la planificación de enfermería de salud pública. Enfoque de investigación cualitativa basada en la perspectiva histórica de la Historia Cultural del historiador francés Roger Chartier, y puesta en funcionamiento en la documentación técnica de la observación, y como una fuente de publicación Informe del Seminario de Salud Pública - Categorías de la OPS surgió después de analizar las necesidades, la planificación y la narrativa de ficción acciones de salud. Resultados: se encontró a través de las relaciones de poder de ficción narrativa, las configuraciones sociales de la clase y el carácter y el contexto de las enfermeras en América Latina. Se concluye que la estrategia del seminario permitió la construcción de propuestas colectivas y conjuntas a partir del intercambio de experiencias entre las enfermeras participantes
Palabras clave: enfermería, historia de la enfermería, salud pública, necesidades y demandas de servicios de salud, política de salud.

\section{RESUMO}

O estudo objetivou discutir os efeitos pretendidos nas enfermeiras latino-americanas legitimados pela estratégia ficcional para o planejamento das ações de enfermagem de saúde publica. Método: pesquisa qualitativa com abordagem histórica fundamentada na perspectiva da História Cultural do historiador francês Roger Chartier, e operacionalizada na técnica da observação documental, tendo como fonte a publicação Informe Del Seminario de Salud Publica - PAHO, após análise emergiram as categorias: necessidades, narrativa ficcional e planejamento de ações de saúde. Resultado: encontrou-se por meio da narrativa ficcional as relações de poder, as configurações sociais e a personalidade da classe e do contexto das enfermeiras latino-americanas. Conclui-se que a estratégia do Seminário possibilitou a construção de propostas coletivas e comuns a partir das trocas de experiências entre as enfermeiras participantes.

Palavras-chave: enfermagem, história da enfermagem, saúde publica, necessidades e demandas de serviços de saúde, política de saúde.

\section{INTRODUÇÃO}

Na década de 1960, os países da América Latina possuíam características peculiares referentes às condições econômicas, políticas e sociais. A distribuição populacional apresentava uma superioridade rural, com a economia essencialmente agrícola e "no plano político vivenciavam a experiência de regimes centra- 
lizadores e autoritários" (Lima, 2002). A urbanização das cidades latino-americanas produziu indigências sanitárias em determinadas capitais, por um crescimento desordenado gerado pelas moradias improvisadas, onde a infraestrutura sanitária era deficiente ou nula e o setor saúde era caracterizado pela elevada incidência de doenças infecciosas que acarretavam uma alta mortalidade infantil e baixa expectativa de vida (Cueto, 2007).

A Organização Pan-Americana da Saúde - OPAS é um dos pioneiros organismos de cooperação internacional na área da saúde e foi criada em dezembro de 1902, na cidade do México durante o evento da "I Convenção Sanitária Internacional das Repúblicas Americanas" (Cueto, 2012). Sua principal atuação deu-se inicialmente por meio da participação nas Conferências Sanitárias Pan Americanas que difundiam as ideias científicas e ações de saúde desenvolvidas pela Organização por intermédio da publicação Boletín de La Oficina Sanitária Pan-Americana (Lima, 2002). A partir de então, principalmente na década de 1960, a OPAS desempenhou uma função de evidência na América Latina, inclusive ao participar ativamente da identificação dos fatores relativos ao desenvolvimento, bens e serviços básicos e qualidade de vida. A princípio esse organismo teve o papel de coibir as doenças transmissíveis pelas grandes circulações portuárias, a exemplo, a febre amarela e a peste bubônica. Progressivamente, verifica-se uma ampliação das ações e do próprio conceito de saúde. Talvez, os fatores mais relevantes a serem acompanhados na história da organização na década de 1960 tenham sido a formulação e aplicação de políticas especificas, além da formação do desenvolvimento da agenda de problemas e adoção de saúde, particularmente nos países da América Latina e Caribe (Lima, 2002).
Historicamente, as políticas públicas e os sistemas e serviços de saúde da América Latina possuíam como particularidade subsidiar a elaboração de planos regionais de saúde, projetos básicos que estabeleciam metas em prol de melhorias na saúde de suas populações e avaliação das condições e hiatos da saúde (Del Seminario de Salud Publica, 2007). Nesse panorama, um programa de destaque foi a Carta de Punta Del Este, documento assinado em agosto de 1961 pelos países membros da Organização dos Estados Americanos (OEA), o qual "continha metas específicas para o combate aos graves problemas de saúde pública que assolavam o continente" (Paiva, 2004), sendo reconhecido como marco político institucional e simbólico na história da saúde pública na América Latina no século XX, além de incluir a saúde como um dos componentes essenciais para o desenvolvimento e a ideia de que o bem-estar resultaria de melhores condições de vida nas nações latino-americanas. Em 1961, a convite da OPAS, foi organizado, por um grupo de enfermeiras latino-americanas de Costa Rica, Guatemala, Honduras, México, Nicarágua, El Salvador e Panamá, o Seminario Del Salud Publica, do qual resultou a publicação denominada Informe Del Seminario en Salud Publica, publicado pela Organizacion Pan-americana de La Salud- OPAS em 1962, que teve finalidade intercambiar ideias e fomentar discussões acerca das experiências de assistência e de educação em saúde das enfermeiras latino-americanas. O Seminario Del Salud Publica ocorreu na cidade de El Salvador, de 5 a 18 de novembro de 1961, para abordar o tema Participação de la enfermera en el planeamiento de servicios de salud del país X. As enfermeiras participantes utilizaram a narrativa ficcional como um recurso lúdico e criativo, para a implementação de um plano assistencial, a partir 
de informações gerais sobre os indicadores de saúde deficientes do país $\mathrm{X}$, fornecidos pela Organização Pan-americana da Saúde (OPAS) durante o seminário.

Após leitura da publicação Informe Del Seminário de Salud Publica surgiu o interesse em estudar o uso da narrativa ficcional para descrever um "país” que apresente um conjunto de necessidades em saúde, suficiente para servir como modelo experimental para uma prática pedagógica para o grupo de enfermeiras latino-americanas.No estudo intitulado "O cinema: outra forma de 'ver' a história", discutem-se as narrativas ficcionais utilizadas no cinema e nos discursos históricos, de modo que distingue-se a narrativa ficcional cinematográfica da narrativa histórica (Junior, 2006). Na primeira, o cinema tem por finalidade contar uma boa história, enquanto a narrativa histórica difere-se por ser utilizada como um instrumento do qual os historiadores se valem para compartilhar com a sociedade os conhecimentos que refletem a memória de uma comunidade em uma determinada época (Junior, 2006).

Assim, cabe elucidar a seguinte questão: Como um grupo de enfermeiras latino-americanas descreveu um plano assistencial a partir das necessidades em saúde encontradas em um "País" ficcional, no Seminario de Salud Publica, promovido pela Organizacion Panamericana de La Salud, em 1962? Dessa forma, delimitamos como objeto de investigação: os efeitos do uso da narrativa ficcional de um 'país' com necessidades em saúde para elaboração de plano assistencial por enfermeiras latino-americanas registrado no Informe Del Seminario de Salud Publica, distribuído pela "Organizacion Panamericana de La SaludOPAS e Organizacion Mundial de La SaludOMS”, em 1962.
Para operacionalizar o estudo, utilizamos os seguintes objetivos: identificar as principais necessidades traçadas durante o Seminario en Enfermería de Salud Publica; analisar a narrativa ficcional como estratégia para elaboração de um plano assistencial de Saúde Publica; discutir os efeitos pretendidos nas enfermeiras latino-americanas legitimados pela estratégia ficcional para o planejamento das ações de enfermagem de saúde publica.

\section{MÉTODO}

Trata-se de uma pesquisa qualitativa de natureza histórica, cujo desenvolvimento foi operacionalizado com o uso da técnica da observação documental, fundamentada na perspectiva da História Cultural do historiador francês Roger Chartier, que permitiu a análise dos os efeitos do uso da narrativa ficcional de um "país" com necessidades em saúde para elaboração de plano assistencial por enfermeiras latino-americanas registrado no Informe Del Seminario de Salud Publica, por meio de estudos de documentos (Aróstegui, 2006). A História Cultural é definida por Chartier como uma abordagem histórica concebida pela reflexão crítica, que era contrária à história convencional, e configuram-se como foco de análise a tríade de conceitos: Representação, Prática e Apropriação (Chartier, 2002). A fonte preferencial desta investigação foi o Informe Del Seminario de Salud Publica, arquivado no acervo do Laboratório de Abordagem Científica da Enfermagem (LACENF) da Escola de Enfermagem Alfredo Pinto da Universidade Federal do Estado do Rio de Janeiro (EEAP-UNIRIO). Os critérios para seleção das fontes foram: a delimitação temporal - o ano de 1962 -, atendo-se à seleção de documentos referentes ao contexto sociopolítico-econômico e com ênfase nas ações de planejamento de en- 
fermagem na saúde publica desenvolvidas na América Latina no entorno desse ano; a divulgação dos informes instituídos pela Organização Pan-americana de Saúde - OPAS, na temática de ações ou modelos de planejamento em enfermagem de saúde pública na América Latina.

A análise dos dados foi realizada pela triangulação dos seguintes materiais: O Informe Del Seminario En Enfermeria De Salud Publica, a interpretação das obras de História Cultural sob a perspectiva de Roger Chartier e as considerações subjetivas dos autores. Dessa análise foram desvendadas três categorias: necessidades traçadas pelas enfermeiras, estratégia ficcional e os efeitos pretendidos em uma comunidade de enfermeiras.

\section{RESULTADOS E DISCUSSÃO}

\section{As necessidades traçadas no Seminario en Enfermeria de Salud Publica.}

Na década de 1960, a OPAS induziu os gestores a adotarem ações de saúde coletiva como meio da sociedade obter benefícios como, por exemplo, mais profissionais de saúde produtivos. Desta forma a noção de saúde vinculou-se à de bem-estar social, pela qual a prevenção e ampliação dos serviços públicos geraria a concepção de saúde como direito individual e coletivo dos cidadãos. E assim, estrategicamente, os governos começaram a enxergar a promoção do bem-estar como alternativa eficaz para minimização de possíveis desigualdades nos modelos assistenciais de saúde da comunidade (Nunes, 2007).

$\mathrm{O}$ Seminario en Enfermeria de Salud Publica sob a fundamentação dessa nova perspectiva de profissionais de saúde produtivos e atuantes, relata as dificuldades e manifestas pelos serviços de saúde, nos quais havia uma precariedade na coordenação das atividades destinadas ao fomento da promoção, proteção e recuperação da saúde.

Inquietado com esse cenário, um grupo de enfermeiras latino-americanas, organizou-se de maneira a agregar todos os conhecimentos teóricos e práticos de enfermeiras do ramo da educação e dos serviços assistências, para que propusessem, através de seus saberes, o desenvolvimento de um programa de Enfermagem em Saúde Pública ou um modelo de planejamento de serviços.

Nas táticas de promoção da saúde, as enfermeiras latino-americanas relatam a necessidade do desenvolvimento de processos de planejamento e gerenciamento direcionados a melhorias das condições de vida e saúde da população do país X. Esses estudos modelos empregados para melhorias da saúde devem pressupor uma abordagem crítica da prevenção, educação em saúde e da promoção da saúde para que o modelo ou manual de ações de planejamento em saúde pública atenda as necessidades demandadas e evidenciadas pela população do país (Santos, 2010).

O debate inicial do seminário teve como tema: La participación de la enfermeira en el planeamiento de servicios de salud del país $X$, o qual foi subdividido em dois temas: 1) fatores que influem no planejamento de serviços (foram traçadas as principais necessidades baseadas nas situações e experiências advindas dos países dessas enfermeiras); 2) planejamento de um serviço de enfermagem (foram implementadas as medidas para o plano de ações de saúde publica) como embasamento para as discussões, questões de saúde pública que foram exploradas e debatidas no seminário.

No país $\mathrm{X}$, a enfermeira chefe da direção geral de saúde pública, convidada pelo diretor 
geral de saúde pública, torna-se membro integrante do comitê de planejamento do plano nacional de saúde, prosseguindo uma investigação deste setor e identificando problemas, como programas de saúde pouco esclarecidos por serem mal redigidos. Logo, os serviços da enfermagem não geram bons resultados, somados ao déficit de profissionais qualificados, seja por limitações orçamentárias ou por preferências a determinados setores, obtendo como consequências insatisfações sobre os serviços prestados. Em nível de titulação, não há incentivo à educação continuada e as demandas de atendimento assistencial médico não suprem as necessidades da população.

A intervenção em prevenção, proteção e promoção de saúde coletiva tem maior probabilidade de êxito quando orientados em bases teóricas e modelos de saúde (Cabrera, 2007), no referido seminário o modelo criado facilitou a visualização das necessidades promovendo nos debatedores aproximação com a temática, permitindo discussões orientadas e pontuais aos profissionais envolvidos nos processos articulados.

Nesta perspectiva, a enfermeira estipula um grupo, constituído por 35 enfermeiras, das quais 27 foram representantes de sete países (distribuídas de acordo com o quadro no 1) e 8 de representantes de Organizações Internacionais (distribuídas conforme o quadro no 2) para colaborar na solução destes, tendo como direcionamento os eixos temáticos: $A$ participação da enfermeira no planejamento de serviços de saúde e o planejamento de um serviço de Enfermagem (Lima, 2002).

Quadro No 1 - Distribuição das enfermeiras participantes no "Seminario en Enfermeria de Salud Publica”, conforme os países. Rio de Janeiro, 2011.

\begin{tabular}{|c|c|}
\hline Países & Enfermeiras \\
\hline Costa Rica & 4 \\
\hline El Salvador & 6 \\
\hline Guatemala & 3 \\
\hline Honduras & 4 \\
\hline México & 2 \\
\hline Nicarágua & 4 \\
\hline Panamá & 4 \\
\hline Total & $\mathbf{2 7}$ \\
\hline
\end{tabular}

Fonte: Informe del seminario en enfermeria de salud publica, 1962.

Quadro No 2 - Distribuição do quantitativo de representantes de Organismos Internacionais no "Seminario en enfermeria de salud publica", conforme os países. Rio de Janeiro, 2011.

\begin{tabular}{|c|c|}
\hline Organizações Internacionais & Representantes \\
\hline Assessoras *OPAS/OMS & 6 \\
\hline Assessoras **AID & 2 \\
\hline Total & 8 \\
\hline
\end{tabular}

Fonte: Dados retirados do Informe del seminario en enfermeria de salud publica, 1962

* OPAS/ OMS - Organização Pan-Americana da Saúde e Organização Mundial da Saúde

${ }^{*}$ AID-Agência Internacional de Desenvolvimento.

Assim, para evidenciar os problemas, as enfermeiras latino-americanas reuniram diversos dados epidemiológicos deficientes para identificar os possíveis determinantes e condicionantes de saúde do país X. A partir da compilação destes dados, elas agruparam os fatores que poderiam interferir no desenvolvimento do país nos seguintes grupos: 1- Informações gerais relacionadas com a geografia, clima, população, cultura, governo e economia; 2- Problemas de saúde e 3- Educação geral e relacionada com a enfermagem. 
As necessidades identificadas embasaram a discussão de metodologias de enfrentamento das dificuldades vivenciadas pelo país ficcional, despertando nos membros integrantes do comitê um pensamento crítico e reflexivo sobre a aplicabilidade de soluções adaptáveis às diferentes realidades.

\section{A narrativa ficcional: Estratégia para elabo- rar o plano assistencial de saúde publica}

As narrativas ficcionais são métodos narrativos que em determinadas circunstâncias contribuem para criar um efeito de verdade, tornando o fato possível de acreditar por conferir ações que estão inseridas no sistema de valores próprios da realidade vivenciada pela sociedade (Rocha, 2011).

É a aproximação da veracidade que contribui para o interesse do público pela leitura desses textos. Assim, conforme esclarece Chartier, a ficção é "um discurso que 'informa' do real, mas não pretende representá-lo nem abonar-se nele" (Chartier, 2009).

Esse artifício, utilizado pelas enfermeiras para auxiliar nesse levantamento de problemas, por meio da articulação e debates de ideias discorridos durante o seminário, possibilitou selecionar elementos/ indicadores que provinham da realidade de um país típico da América Latina, que se deparasse com características similares ao país $\mathrm{X}$, já que a apresentação de 'verdade' se faz a partir do emprego sistemático de elementos próprios a esse tipo de narrativa, a ficcional. (Rocha, 2011).

É o que ocorre na introdução ao seminário quando trás o elemento tempo, com sua definição precisa de um determinado contexto espacial. A narrativa, porém, não se fixa apenas na exposição e na descrição do perfil do país $\mathrm{X}$. O documento também narra os fatos tendo como ponto referencial as impressões das en- fermeiras latino-americanas. Elas promovem um diálogo direto com o leitor ao apresentar a versão dos fatos, expondo os problemas existentes nos serviços de Saúde Pública.

Ao ilustrar o caso ficcional representado pelas enfermeiras, a narrativa não tem caráter ilusório e não é construída por casos imprevistos ou aleatórios. A base do estudo de caso ficcional é estruturada conforme o contexto sociopolítico e econômico correspondente à época de 1960, indo ao encontro dos avanços e aos esforços coletivos e políticos, a exemplo dos Planos Regionais de Saúde presentes no contexto do real vivenciado pela década de 1960 (Del Seminario de Salud Publica, 2007).

Essas formas de retratar a realidade objetivam empregar uma metodologia pedagógica para diferenciar o que necessita ser alterado ou exaltado em uma comunidade que enfrenta uma circunstância que precisa de modificação.

\section{O efeito da leitura ficcional na comunidade de enfermeiras para o planejamento de ações de Saúde Publica}

Elege-se a leitura como um mecanismo/ ferramenta primordial de instrução e correção de irregularidades, retendo e desempenhando função crucial no desenvolvimento do intelecto e na formação de um caráter e da moral ficcional. "A leitura não é um ato simples, mas sim problemático, que exige um leitor atento e vigilante a quem cabe a construção do sentido" (Rocha, 2011). Ainda que as respostas a essa leitura sejam variadas, elas partem de dispositivos textuais operados pelo autor com a finalidade de levar o leitor a ler de certa forma e convoca a interação e interpretação do mundo (Chartier, 2003).

Há também a compreensão de que a produção de sentido como uma relação dialógica entre as propostas do texto e as categorias in- 
terpretativas de seus leitores (Chartier, 2003). No entanto, certas recorrências na interpretação nos possibilitam organizar um pensamento ou uma estratégia comum.

O documento produzido pela Organização Pan-americana da Saúde instaurou uma orientação para esclarecer e solucionar as principais necessidades, ou seja, os fatores determinantes e condicionantes identificados pelas enfermeiras latino-americanas.

Elas apropriaram-se da narrativa ficcional e desenvolveram, com soluções imediatas, um programa de planejamento de Enfermagem Materno-infantil, visto que diante de tamanhas deficiências não seria viável banir todos os problemas de uma única vez. Dessa forma, as enfermeiras elegeram os cuidados ou ações características da atenção primária que atingissem a base do problema e semeassem informações pertinentes à prevenção. Para que esses incidentes não se agravassem ainda mais, elas programaram metas de curto e de longo prazo com atividades que deveriam ser operacionalizadas, além de estudarem os fatores que poderiam interferir em seu desenvolvimento, agrupando-os em: informações gerais relacionadas à geografia, clima, população, cultura, governo e economia; problemas de saúde e Educação geral e relacionada, em especial, com os profissionais de enfermagem.

Com base na análise e discussão dos indicadores epidemiológicos do estudo realizado pelas enfermeiras, a morbidade e mortalidade infantil foram eleitas como os indicadores mais preocupantes, o que as levaram a criar um plano ou um programa materno-infantil.

Levando em consideração os problemas sanitários do país e os recursos dos profissionais, pensou-se na melhor utilização destes nos diferentes serviços existentes, de acordo com sua distribuição e preparação, para ela-

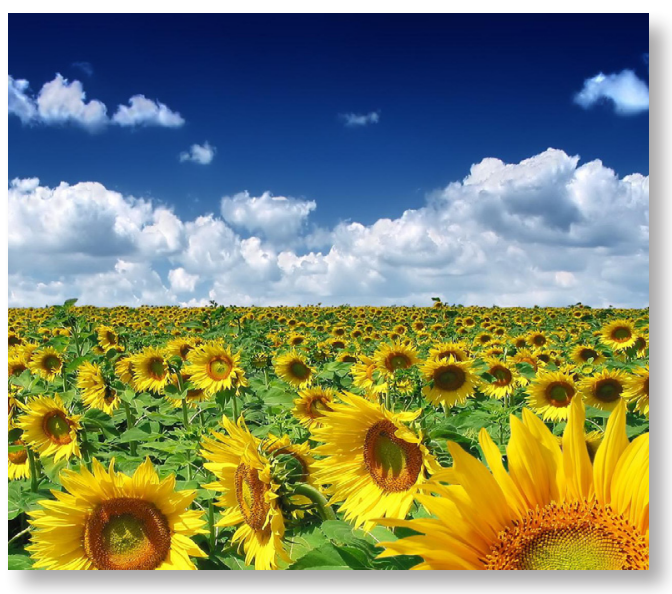

boração das atividades nos diversos aspectos analisados. Para iniciar essa segunda etapa, levou-se em consideração o objetivo geral do programa materno-infantil e estabeleceram-se a filosofia e objetivos referentes à enfermagem.

Como o programa de enfermagem é parte integrante do programa nacional de saúde, consideraram-se as ações de enfermagem que tinham relação com a nutrição, controle de enfermidades transmissíveis, saneamento ambiental, educação para a saúde e alguns dados estatísticos do país X, observamos a instauração de uma medida simples e viável para a melhoria de condições básicas de saúde.

Os efeitos ou desdobramentos da leitura pelas enfermeiras latino-americanas no Informe del seminario em enfermeria de salud publica foram evidenciados a partir da interpretação antropológica dessas comunidades, recurso imprescindível para pensar: a construção do vínculo social e a subjetividade (Almeida, 2011).

A associação do que foi compreendido com a leitura e o que apreendemos, elaborado de forma mais organizada conforme a representação do pensamento que aplicamos em nossas experiências, gera uma identidade. Essa agregação é definida como signo imaginativo (Duarte, 2011). 
Esse signo imaginativo auxiliou as enfermeiras latino-americanas a empregarem outro conceito importante o processo civilizador, não apenas como uma educação do corpo, da higiene e da etiqueta, mas como descreveu Nobert Elias e Foucault que é a educação pela linguagem materialmente transmitida, a partir da leitura-apropriação, o "retorno sobre si mesmo" (Rocha, 2011). Na experiência dada aos leitores do "Seminario en Enfermeria de Salud Publica" evidenciou-se a integração do signo imaginativo com a ficção, já que o aperfeiçoamento do processo educativo em saúde requer o desenvolvimento de metodologias participativas comparadas a situações concretas (Santos, 2010), adequando-se tempo, narrativas coletivas e recursos pedagógicos às condições de equalização de oportunidades de aprendizagem e empoderamento individual e comunitário.

\section{CONCLUSÕES}

$\mathrm{O}$ presente documento Informe Del Seminario de Salud Publica, fruto do Seminário "Participación de la enfermera en el planeamiento de servicios de salud del país X”, exercita, através da narrativa ficcional, as relações de poder, as configurações sociais e a personalidade da classe e do contexto estudado. A noção de representação sob a perspectiva de Roger Chartier auxiliou a compreensão das características coletivas comuns de um grupo de enfermeiras participantes do Seminário, que, através do incentivo aos debates fomentados pela Organização Pan-Americana da Saúde, promoveram discussões que gerassem medidas eficientes e flexíveis para a correção dos hiatos da saúde em seus países de origem.

Essa abordagem acrescentou a transformação da leitura de uma ficção em método de auxílio e desenvolvimento de saberes sobre medidas de atuação profissional. Por meio de um manual de atenção materno-infantil, buscou-se nas leitoras enfermeiras ampliar suas expectativa em solucionar as inúmeras deficiências habituais de forma condizente e cabível ao contexto sociopolítico e econômico da América Latina na década de 1960.

Neste estudo, tentou-se retomar as condições e dificuldades deparadas no que reflete as situações assistências de Saúde Publica nesse país ficcional, além de explanar a definição da narrativa ficcional e da leitura de apropriação das enfermeiras latino-americanas, ou seja, como elas empregaram a associação da compreensão da leitura ficcional e das vivências em suas experiências profissionais e pessoais. Possibilitando a sistematização da assistência coletiva através do planejamento de saúde publica.

\section{REFERÊNCIAS}

- Almeida, P. F., Fausto, M. C. R. Giovanella, L. (2011). Fortalecimento da atenção primária à saúde: estratégia para potencializar a coordenação dos cuidados. $\mathrm{Rev} \mathrm{Pa-}$ nam Salud Publica. Recuperado de: http://www.scielosp. org/scielo.

- Aróstegui, J. (2006). A Pesquisa Histórica: teoria e método. Bauru (SP): Editora Edusc.

- Auer, A., Guerrero E. J. E. (2011). La Organización Panamericana de la Salud y la salud internacional: una historia de formación, conceptualización y desarrollo colectivo. Rev Panam Salud Publica. Recuperado de http:// www.scielosp.org/scielo.

- Cabrera A. G. A. (2007). Uso de teorías y modelos en artículos de una revista latinoamericana de salud pública, 2000-2004. Rev. Saúde Pública, 963-969. Recuperado de http://www.scielosp.org/scielo.

- Chartier, R. (2009). A história ou a leitura do tempo. $1^{\mathrm{a}}$ ed. Belo Horizonte (BH): Editora Autêntica.

- Chartier, R. (2003) Formas e sentidos cultura escrita: entre distinção e apropriação. $1^{\text {a }}$ Ed. São Paulo: Mercado de 


\section{ᄃultura de las Cuidados}

Letras; Associação de leitura do Brasil (ALB), Coleção Histórias de Leitura

- Chartier, R. (2002). A História Cultural entre práticas e representações. $2^{\text {a }}$ ed. Lisboa (LIS): Difusão Editorial.

- Cueto, M. (2007). O valor da saúde: história da Organização Pan-Americana da Saúde. $1^{\text {a }}$ ed. Rio de Janeiro (RJ): Editora Fiocruz.

- Duarte, G. M. B., Ortega, A. M. L., Mora, R. L. H., Coromoto, F. N. (2011). Un modelo de atención en salud al pueblo Wayúu en la frontera colombo-venezolana. Rev Panam Salud Publica, p. 272-278. Recuperado de http:// www.scielosp.org/scielo.

- Junior, R. A. (2006). O cinema: outra forma de "ver" a história. Revista Iberoamericana de Educación, 25, 1-12. Recuperado de http://www.rieoei.org/1244.htm.

- Lima, N.T. (2002). Caminhos da Saúde Pública no Brasil. $2^{\text {a. }}$ ed. Rio de Janeiro (RJ): Editora Fiocruz.

- Nunes, E. S. N. (2007). Resenha de O valor da saúde: his- tória da Organização Pan-Americana de Saúde de Marcos Cueto. Rev Bras História,28, 597-60. Recuperado de http://www.scielo.br/pdf/rbh/v28n56/20.pdf, baixado em 27 de Nov de 2011.

- Organización Panamericana de la Salud. Salud em las Américas (2007). 1 1 Ed.Washington, DC: PAHO; ( Publicación Científica y Técnica 622; 1 vol.).

- Paiva C. H. A. (2004). A Organização Pan-americana de saúde (OPAS) e a reforma de recursos humanos na saúde da América Latina (1960-70). Recuperado de observatoriohistoria.coc.fiocruz.br/local/File/opas.pdf

- Rocha, J. C. Organizador. (2011). Roger Chartier a força das representações: história e ficção. $1^{\text {a }}$ Ed. Santa Catarina: Argos.

- Santos, L. M., Oliveira, E. M., Crepaldi, M. A., Aurélio, R. M. (2010). Atuação dos coordenadores de grupos de saúde na rede docente assistencial. Rev. Saúde Pública, 34, 177-184. Recuperado de http://www.scielo.br/scielo.

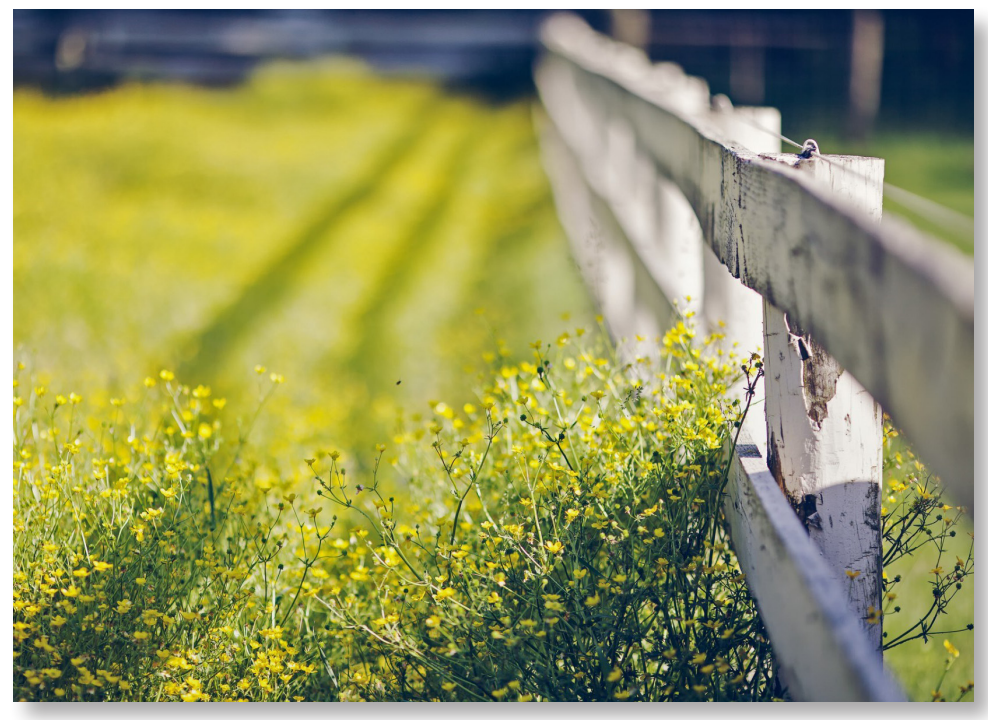

